\title{
The Twin Towers Cluster in Torah Codes
}

\author{
Eliyahu Rips \\ Institute of Mathematics \\ Hebrew University, Givat Ram \\ Jerusalem 91904, Israel \\ rips@cc.huji.ac.il
}

\author{
Art Levitt \\ Torah Code Research Group \\ Jerusalem, Israel \\ artlevitt23@yahoo.com
}

\begin{abstract}
In this paper we describe a Torah Code experiment which:

- Focuses on a famous contemporary event.

- Has a simple and explicit data collection.

- Has considerably simpler measurements than in previous Torah Code experiments.

The pattern we study here is a cluster of related key words in the form of ELSs in a single table. The cluster that we discover contains a subset of all key words obtained in the data collection. We account for the choices needed to determine this subset using the Bonferroni inequality. Our protocol involves some other explicit choices, accounted for in a similar way. The final p-value is less than $5.14 \times 10^{-5}$.
\end{abstract}

\section{Introduction}

\subsection{Background}

Torah Code research studies the evidence that the text of Torah (the first five books of the Hebrew Bible) contains a hidden encoded text that describes world reality (cf. [5], Ch. 5). The clustering of equidistant letter sequences (ELSs, defined below) for key words related to a certain topic is one of the effects providing such evidence (see discussion in [7], p.429).

The event we have chosen for the present experiment is the Twin Towers attack. Since a reasonable choice of key words is not unique, we used the key words appearing in the actual newspaper headlines reporting the attack.

\subsection{Basic notions}

To form an ELS from a text, we ignore all punctuation and inter-word spaces. For example, the ELS "tin tops" can be found starting with the first " $t$ " in the word "punctuation" in the preceding sentence, and using a skip of +4 (that is, counting forward every 4 letters from the starting position).

To graphically present ELSs, we use tables where the text is arranged with rows of equal length, with all spaces removed (see Figure 2, where the rows are of length 36 ). On such a table, the letters of an ELS form a straight line, unless the ELS wraps around the table. In particular, an ELS with skip 36 will appear vertically on the table.

By a cluster we mean a grouping of ELSs, an example of which is also seen in Figure 2.

\section{Description of the experiment}

\subsection{Analysis of the headlines}

Figure 1 displays the headlines from a major Israeli newspaper, Maariv, on September 12, 2001. The English translation follows:

"America is Burning; The HORROR, the SHOCK, the VICTIMS; Impossible to Believe. The biggest TERROR ATTACK in history happened yesterday. Two hijacked AIRPLANES exploded into the TWIN TOWERS in New York and caused their collapse. A third AIRPLANE exploded into the Pentagon. A fourth crashed in Pennsylvania. First estimates: between 10,000 and 25,000 DEAD; more than 25,000 trapped in the RUINS. President Bush flew to the headquarters of atomic war in Nebraska. All airports in the U.S. were closed; the borders with Canada and Mexico were locked. The immediate suspect: Osama bin Laden. The explosions in the capitol of Afghanistan: U.S.: it is not us. National day of MOURNING in Israel Peres: 'it is like an atomic bomb'; thousands of Palestinians celebrated in the streets."

To keep our experiment as simple as possible, we concentrate on the nouns in their basic form.

We divide the nouns into four categories.

The first category contains the primary (most relevant) nouns: TWIN TOWERS, AIRPLANE, AIRPLANES, 


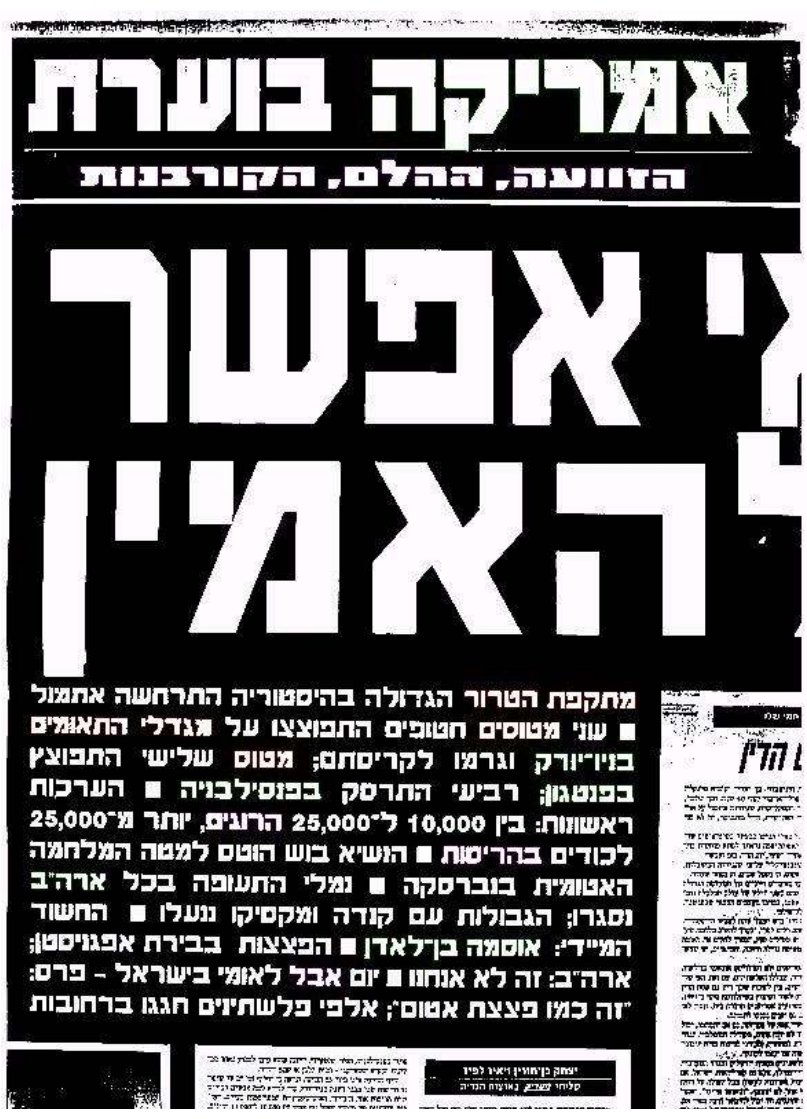

Figure 1. The Twin Towers headlines as they appeared in the Hebrew Newspaper "Maariv", 12 September 2001.

\section{TERROR ATTACK.}

The second category contains the relevant proper nouns not included in the first category: America, New York, Pentagon, Pennsylvania, US, Osama bin Laden.

The third category contains the secondary (other relevant) nouns: HORROR, SHOCK, VICTIMS, [the] DEAD, RUINS, MOURNING.

The fourth category contains all other nouns, not highlighted.

For simplicity, we follow the spelling of the newspaper exactly as it appears, and we delete the definite article whenever possible (however, we cannot delete it in the Twin Towers for grammatical reasons).

\subsection{Measuring the clusters}

In this study, we have chosen the two simplest ways to measure the compactness of a cluster of ELSs. First, when considering Torah Codes as a two-dimensional phe- nomenon, we use the area of the smallest rectangular window in the Torah Code table containing all of the ELSs. The second approach takes a one-dimensional view, and uses the length of the shortest segment of the text containing all of the ELSs. ${ }^{1}$

To estimate the significance of the clusters, we compare them to analogous clusters in a control population. For this, we use the ELS Random Placement (ERP) method developed by Professor Haralick, and we refer to [2], section 14 for precise definitions. In summary, each member of the control population is created by randomly translating the locations of the original ELSs found in the Torah, and preserving their skips.

Using this protocol, we are able to test the Null Hypothesis of no Torah Code effect against the Alternative Hypothesis that there are one or more unusually compact $1 \mathrm{D}$ or $2 \mathrm{D}$ clusters containing ELSs of each of the key words.

\subsection{The software and parameter settings}

We use the software provided by Professor Haralick for the one-dimensional and two-dimensional measurements, with parameters set as detailed in section 3 .

\subsection{The experimental design}

The pattern that we are trying to detect is the existence of unusually compact clusters. Since not all of the key words may be present in a cluster (for example, the encoded text might use words other than the newspaper key words), we must check for the clustering of subsets of our sets of the key words.

Our protocol allows for searching in the whole Torah or in any of the five books of Torah separately.

We use both one-dimensional and two-dimensional measurements.

We will account for all of the above choices in sections 2.6 and 2.7 .

\subsection{Outcome of the experiments}

\subsubsection{The experiment with the primary nouns.}

Four of the six primary nouns, TWIN TOWERS, AIRPLANE, ATTACK appear in the Book of Numbers in a very compact cluster of size $13 \times 18$ (see Figure 2).

\footnotetext{
${ }^{1}$ The compactness measure developed in [7] applied to the meetings of pairs of ELSs. Instead of generalizing it for clusters of ELSs, we preferred to adopt the approach of Professor Haralick ( [2], [3]). While for the onedimensional measurement ( [3], section 4), the length of the text segment is the only option, Professor Haralick uses a variety of measures for the rectangular window, of which we have chosen area, the simplest one (see [2] section 9.3).
} 
Professor Haralick's two-dimensional program found 10 clusters of smaller area for an ERP text population of size 100 million, giving the significance value $1.05 \times 10^{-7}$.

\subsubsection{The experiment with the proper nouns.}

\section{Airplane Attack the Twin Towers}

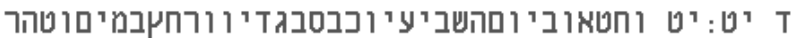

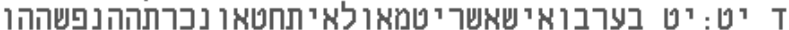

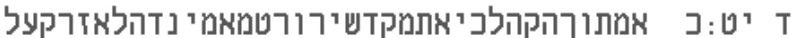

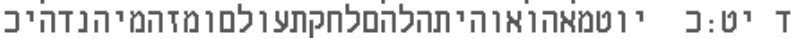

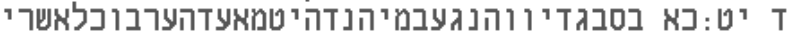

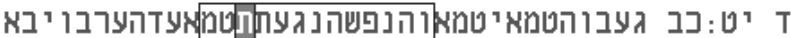

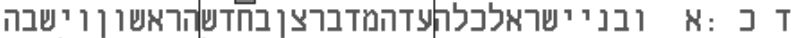

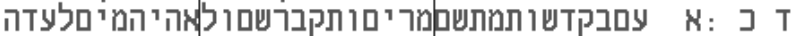

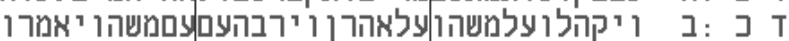

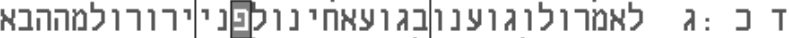

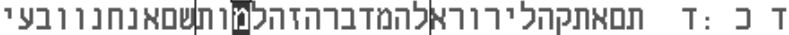

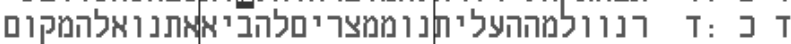

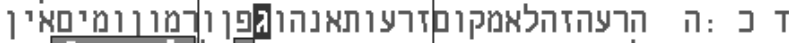

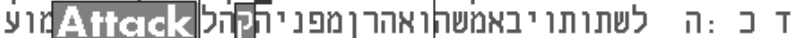

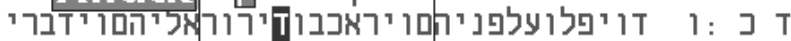

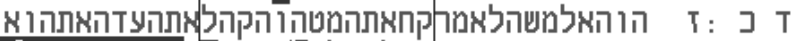

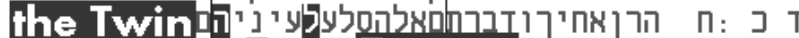

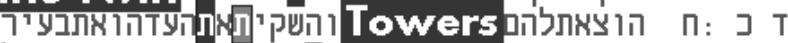

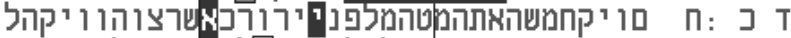

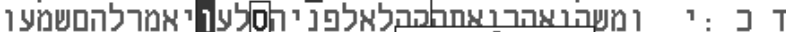

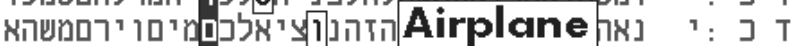

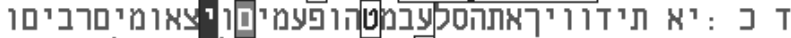

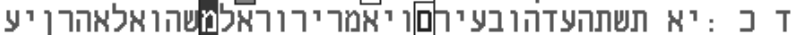

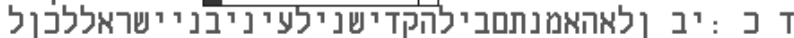

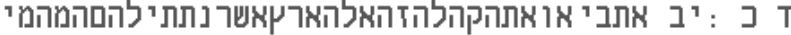

Figure 2. The cluster of the primary nouns, with English translations inserted for the reader's convenience

No significant cluster of ELSs was found for the proper nouns.

\subsubsection{The experiment with the secondary nouns.}

Five of the six secondary nouns, HORROR, SHOCK, [the] $D E A D$, RUINS, MOURNING cluster in the same location as the primary nouns (see Figure 3 ).

In order to ensure the same location for the second cluster, we included the Twin in the second search. Professor Haralick's one-dimensional program found 110 clusters of shorter text length for an ERP text population of size 10 million, giving the significance value $1.105 \times 10^{-5}$.
Ruins 36

T יט:יט וחטאוביוםהשביעי וכבסבגדיוורחקיפמיםוטהר

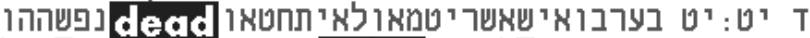

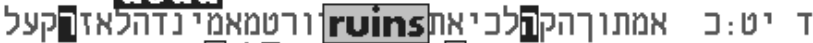

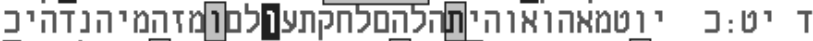

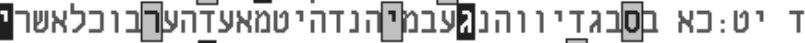

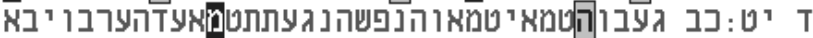
ד כ :

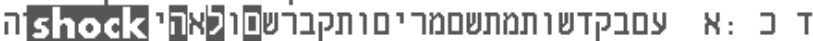

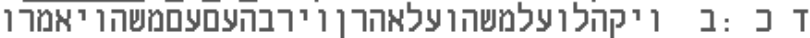

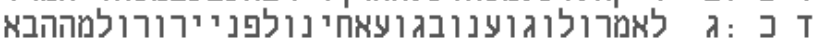

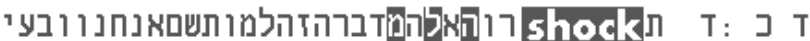

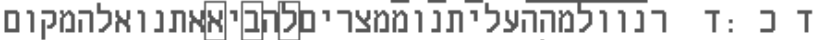

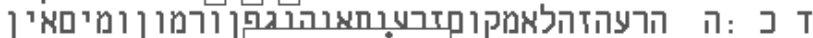

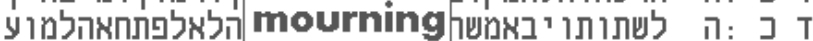

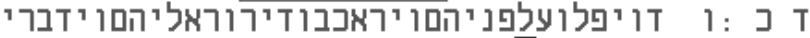

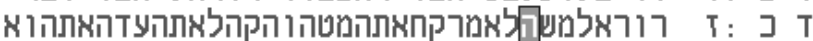

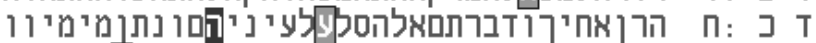

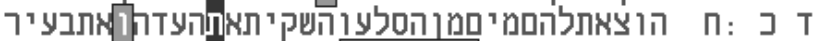

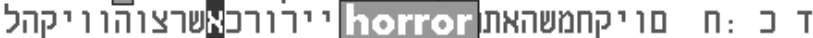

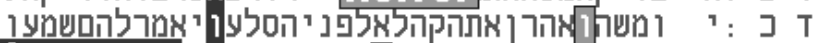

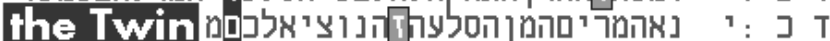

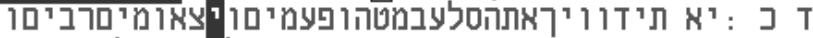

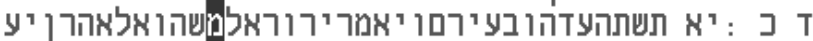

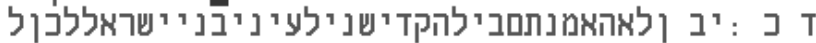

\section{Figure 3. The cluster of the secondary nouns in the same location}

\subsection{The choice factors}

\subsubsection{General choices.}

A factor of 3 accounts for our choice of the Maariv headlines - there are two other major Hebrew newspapers in Israel (Haaretz and Yedioth).

A factor of 6 accounts for the choice of searching in any of the five books of Torah, or in the entire Torah. Our choice was the Book of Numbers.

\subsubsection{The choices for the experiment with the primary} nouns.

1. A factor of 64 accounts for the 6 binary choices to include or exclude each of the primary nouns cited in the headlines. We have chosen 4 out of 6 nouns as indicated in 2.5.1.

2. A factor of 2 accounts for the choice of onedimensional or two-dimensional measurement. 


\subsubsection{The choices for the experiment with the sec- ondary nouns.}

1. A factor of 64 accounts for the 6 binary choices to include or exclude each of the secondary nouns cited in the headlines. We have chosen 5 out of 6 nouns as indicated in 2.5.3.

2. A factor of 2 accounts for the choice of onedimensional or two-dimensional measurement.

\subsubsection{The choice of the subset of experiments.}

A factor of 8 accounts for the choices to include or exclude each of the three experiments (with the primary nouns, with the proper nouns and with the secondary nouns). Our choice is to include the experiment with the primary nouns and with the secondary nouns.

\subsection{Adjustments}

In this section, we apply the Bonferroni inequality and Fisher statistics.

For the experiment with the primary nouns, we multiply the significance value $1.05 \times 10^{-7}$ by factors 64 and 2 from 2.6.2 and obtain the value $1.34 \times 10^{-5}$.

For the experiment with the secondary nouns, we multiply the significance value $1.105 \times 10^{-5}$ by factors 64 and 2 from 2.6.3 and obtain the value $1.41 \times 10^{-3}$.

Applying the Fisher statistic [1] to these values, we obtain $3.57 \times 10^{-7}$.

Accounting for factors 3, 6 and 8 from 2.6.1 and 2.6.4, we finally obtain the p-value $5.14 \times 10^{-5}$ (see [3], section 2.2 for more details on the Bonferroni bound). We therefore reject the Null Hypothesis in favor of the Alternative Hypothesis.

\section{Parameter settings}

The only parameter for the one-dimensional measurement is the expected number of ELSs. For the twodimensional measurement, the maximum row and column skips on the cylinder must also be set (see [2], sections 4, 5 and 6 for skip and resonance specifications).

In WRR [7], the expected number of ELSs was set to 10. WRR relates to an ELS a set of cylinder sizes in such a way that the row skip of this ELS on the corresponding cylinders does not exceed 10 and the column skip does not exceed 5 (page 434).

Later analysis of the data has shown that the value 5 for the row skip is more appropriate (see [6], tables 6 and 7).

In view of the above, we retain the expected number of ELSs $=10$. We set the maximum row skip and column skip to 5. Notice that unlike WRR, the restrictions enforced by row skip and column skip apply to all ELSs in the table.

The above setting is our choice, but, for the record, if we follow [3], section 4, and set the maximum row skip and column skip $=10$, then for the cluster of primary nouns we obtain the value $3.0 \times 10^{-7}$ on a control population of 25 million. If we set the row skip $=10$ and the column skip $=5$, following WRR, we obtain the value $7.0 \times 10^{-8}$ on a control population of 50 million.

In our protocol, despite the fact that we know in advance that the one-dimensional measurement does not give interesting results for ELSs with large skips, and the current implementation of the two-dimensional measurement does not capture the clusters when the ELSs wrap around the cylinder, in every instance we tried both measurements and accounted for both of them.

\section{Discussion}

The headlines on 12 September, 2001, reflect the early perspective, in which bin Laden was only a plausible suspect. In a later perspective, he became the main culprit, which would justify upgrading the keyword bin Laden from the list of proper names to the list of major keywords. In this case, the outcome would be $9.84 \times 10^{-5}$.

In conclusion, the current results, together with several other studies cited in [4], reinforce the evidence for Torah Codes.

\section{Acknowledgments}

We wish to thank Rabbi Earl David and Yuri Pikover for their generous support; and Harold Gans and Professor Robert Haralick for their valuable advice.

\section{References}

[1] R. Elston. On Fisher's method of combining $p$-levels. Biometrical Journal, 33:339-345, 1991.

[2] R. M. Haralick. Basic concepts for testing the Torah Code hypothesis. In Proceedings of the 18th International Conference on Pattern Recognition, August 2006.

[3] R. M. Haralick. Testing the Torah Code hypothesis: The experimental protocol. In Proceedings of the 18th International Conference on Pattern Recognition, August 2006.

[4] A. Levitt. Torah Codes primer and survey of the latest research. http://www.torahcodes.net.

[5] Rabbi Eliyahu of Vilna. Commentary on Safra D'Tsniusa. Vilna, 1882. [in Hebrew].

[6] D. Witztum and Y. Beremez. MBBK's "Study of Variations". http://www.torahcodes.co.il/variat/var_eng.htm.

[7] D. Witztum, E. Rips, and Y. Rosenberg. Equidistant letter sequences in the book of Genesis. Statistical Science, 9(3):429438, August 1994. 